\title{
Defining the Optimal Time of Adaptive Replanning in Prostate Cancer Patients with Weight Change during Volumetric Arc Radiotherapy: A Dosimetric and Mathematical Analysis Using the Gamma Index
}

\author{
Hoon Sik Choi, ${ }^{1}$ Guang Sub Jo, ${ }^{1}$ Jong Pyo Chae, ${ }^{1}$ Sang Bong Lee, ${ }^{1}$ \\ Chul Hang Kim, ${ }^{1}$ Bae Kwon Jeong, ${ }^{2,3}$ Hojin Jeong, ${ }^{2,3}$ Yun Hee Lee, ${ }^{2,3}$ In Bong Ha, ${ }^{2}$ \\ Ki Mun Kang, ${ }^{2,3}$ and Jin Ho Song ${ }^{1,3}$ \\ ${ }^{1}$ Department of Radiation Oncology, Gyeongsang National University School of Medicine and Gyeongsang National University \\ Changwon Hospital, Changwon 51472, Republic of Korea \\ ${ }^{2}$ Department of Radiation Oncology, Gyeongsang National University School of Medicine and Gyeongsang National University \\ Hospital, Jinju 52727, Republic of Korea \\ ${ }^{3}$ Institute of Health Science, Gyeongsang National University, Jinju 52727, Republic of Korea \\ Correspondence should be addressed to Jin Ho Song; cowtree@gnuh.co.kr
}

Received 7 September 2017; Revised 24 October 2017; Accepted 6 November 2017; Published 18 December 2017

Academic Editor: Giancarlo Ferrigno

Copyright (C) 2017 Hoon Sik Choi et al. This is an open access article distributed under the Creative Commons Attribution License, which permits unrestricted use, distribution, and reproduction in any medium, provided the original work is properly cited.

\begin{abstract}
We evaluated the changes in the dose distribution of radiation during volumetric arc radiotherapy (VMAT), to determine the right time for adaptive replanning in prostate cancer patients with progressive weight (WT) changes. Five prostate cancer patients treated with VMAT were selected for dosimetric analysis. On the original computed tomography images, nine artificial body contours were created to reflect progressive WT changes. Combined with three different photon energies (6,10, and 15-MV), 27 comparable virtual VMAT plans were created per patient. The dosimetric analysis included evaluation of target coverage $\left(D_{95 \%}, D_{\max }\right)$, conformity index, homogeneity index, and organs at risk doses. The dose differences among the plans were determined using the gamma index analysis and were compared with the dosimetric analysis. Mean $D_{95 \%}$ became lower than $98 \%$ when body contour expanded by $2.0 \mathrm{~cm}$ or more and $D_{\max }$ became higher than $107 \%$ when body contour contracted by $1.5 \mathrm{~cm}$ or more in $10-\mathrm{MV}$ plans. This cut-off values correlated well with gamma index analysis results. Adaptive replanning should, therefore, be considered if the depth of body contour becomes $1.5 \mathrm{~cm}$ smaller (WT loss) or $2.0 \mathrm{~cm}$ larger (WT gain) in patients treated by VMAT with 10-MV photons.
\end{abstract}

\section{Introduction}

The goal of radiotherapy (RT) is to maximize tumor control while minimizing damage to the surrounding normal tissue [1]. To achieve this goal, conventional RT techniques have been replaced with more sophisticated delivering methods, such as intensity-modulated radiotherapy (IMRT) and volumetric modulated arc radiotherapy (VMAT), which can create steeper dose gradients between the tumor and normal tissue [2-5]. However, if these techniques are not supported by image-guided radiotherapy (IGRT) which uses various inroom imaging devices, the steep dose gradient can increase the risk of missing the target $[6,7]$. With the aid of IGRT techniques such as the in-room cone-beam computed tomography (CT), it is possible to study the set-up errors and the anatomical changes during the RT session. However, it is difficult to determine if the observed anatomical changes are significant enough to consider a new adaptive radiotherapy (ART) plan [8-10].

Prostate cancer is the most common cancer in men, and RT is often used for the treatment of this cancer [11, 12]. Due to the radiobiological features of prostate cancer, often higher doses of RT are required for long durations. However, since the rectum and bladder are in proximity to 


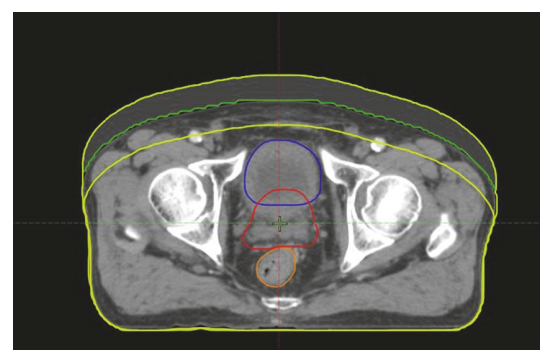

(a)

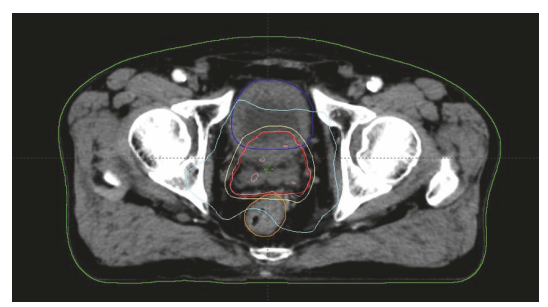

(c)

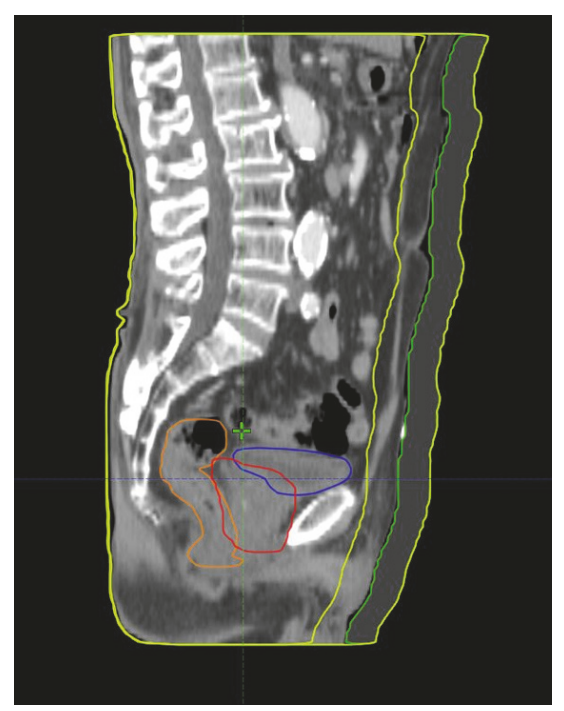

(b)

FIGURE 1: The changes in body contour and target contour. (a) Axial image and (b) sagittal image. The planning target volume (PTV) is shown in red, bladder in blue, and rectum in orange. (c) Dose distribution: the isodose lines are 100\% (pink), 90\% (yellow), and 50\% (sky blue), respectively.

the prostate gland, a very high level of precise ART technique is essential $[13,14]$. For ART, various anatomical changes must be considered, and a change in body weight (WT) is one of them. WT changes are often observed in prostate cancer patients due to various reasons such as dehydration and loss of appetite or as side effects of hormone therapy $[15,16]$. Smith et al. [15] reported that body WT of patients receiving androgen deprivation increased by $2.4 \pm 0.8 \%$. These body WT and body contour changes could affect the radiation dose distribution. Chow and Jiang [16] reported that a $2 \mathrm{~cm}$ decrease in contour depth, caused by the patient WT loss, could increase the dose to the target and normal organs by more than $5 \%$. Since WT changes in every patient vary, individualized ART is needed. However, there are no standard criteria for the optimal time to consider ART.

Therefore, in this study, we calculated and analyzed the dosimetric changes for the targets and organs at risk (OAR) as the patient's WT changed. With the addition of the gamma index, which is usually used to quantify the quality of the delivered calculated plans, we have suggested criteria which will help in deciding the optimal time to consider ART in prostate cancer patients with WT changes.

\section{Materials and Methods}

2.1. Patients and Radiotherapy. Five prostate cancer patients treated at the Gyeongsang National University Changwon Hospital between July 2016 and May 2017 were selected for the study. All patients were treated by VMAT due to lowto intermediate-risk (on the basis of the National Comprehensive Cancer Network) prostate cancer. All patients were immobilized in the supine position with an empty rectum and full bladder. CT images were taken and imported into the Eclipse treatment planning system Version 13.7 (Varian Inc.,
Sunnyvale, CA, USA). The targets and OAR were contoured on these CT images. The prostate and seminal vesicle were delineated as the gross tumor volume (GTV). The clinical target volume (CTV) was defined to be the same as the GTV. The planning target volume (PTV) was created by expanding the CTV by $5 \mathrm{~mm}$ except $3 \mathrm{~mm}$ posteriorly. The rectum and bladder were delineated as OAR. The VMAT plans were made using double 360-degree photon arcs with high definition 120-leaf multileaf collimator from the Varian TrueBeam (Varian Medical Systems, Palo Alto, CA, USA). All patients were prescribed a dose of $78 \mathrm{~Gy}$ in 39 fractions. The VMAT plan was normalized so that $95 \%$ of the PTV received more than $100 \%$ of the prescribed dose. Based on the QUANTEC (Quantitative Analyses of Normal Tissue Effects in the Clinic) guidelines radiation exposure of the rectum was limited so that $V_{60 \mathrm{~Gy}}<50 \%, V_{65 \mathrm{~Gy}}<35 \%$, and $V_{70 \mathrm{~Gy}}<25 \%$ $\left(V_{x \mathrm{~Gy}}\right.$ : the percentage of the organ volume receiving $x$ Gy or more). Similarly, the bladder exposure was limited so that $V_{65 \mathrm{~Gy}}<50 \%$ and $V_{70 \mathrm{~Gy}}<35 \%$ [17]. This study was approved by the Institutional Review Board (IRB) of the Gyeongsang National University Changwon Hospital (IRB 2017-08-012).

\subsection{Body Contour Changes and Virtual Treatment Planning.} The body contours were artificially contracted and expanded in the conventional CT images from $-2.0 \mathrm{~cm}$ to $2.0 \mathrm{~cm}$, by $0.5 \mathrm{~cm}$ increments (Figure 1), to mimic the patients' WT changes. Bones and internal organs were kept in the same position. While the artificially expanded regions were assigned a CT density based on the surrounding soft tissue, the contracted region was replaced by the air density value. Nine different CT sets were prepared for each patient, which included the original CT scan, four expanded CT sets mimicking WT gain, and four contracted CT sets mimicking WT loss. For comparison, adaptive VMAT plans 
were made using these CT sets for each patient, while keeping all other planning parameters, such as the target volume, OAR, prescription dose, beam geometry, and dosevolume optimization criteria same as those in the original VMAT plan. The adaptive VMAT plans were made for three different photon energy changes (6-MV, 10-MV, and 15-MV). Consequently, a total of 27 comparable VMAT plans (on 9 different CT sets with 3 different energies) were made per patient.

2.3. Dosimetric Analysis. By analyzing the dose-volume histogram (DVH), each VMAT plan was compared in terms of PTV coverage, conformity index (CI), homogeneity index (HI), and doses to the rectum and bladder. PTV coverage was analyzed using the terms $D_{95 \%}\left(D_{x \%}\right.$ : the dose to $x \%$ of the volume) and $D_{\max }$ for PTV. The recalculated plan was considered unacceptable, if the PTV $D_{95 \%}$ is lower than $98 \%$ or the PTV $D_{\max }$ is higher than $107 \%[18,19]$. The plan was also considered unacceptable, if the dose to the rectum and bladder exceeded the QUANTEC recommended dose constraints.

The conformity and homogeneity indices for all the plans were calculated using the following formula $[20,21]$ :

$$
\text { Conformity index }=\frac{V_{\mathrm{RI}}}{\mathrm{TV}} \text {, }
$$

where $V_{\mathrm{RI}}$ is defined as the volume encompassed by the prescription isodose and TV is the target volume. The optimal value is 1 which corresponds to an ideal conformation. A CI larger than 1 indicates that the irradiated volume is greater than the target volume and includes some normal tissues, while CI lower than 1 indicates that only a part of the target volume is getting irradiated [20].

$$
\text { Homogeneity index }=\frac{D_{5 \%}}{D_{95 \%}} \text {. }
$$

$D_{5 \%}$ and $D_{95 \%}$ are the minimum doses to $95 \%$ and $5 \%$ of the target volume, respectively. The ideal value is 1 and an increase of inhomogeneity results in an increased $\mathrm{HI}$.

2.4. Gamma Index Analysis. Gamma index is a concept to calculate the difference between the calculated plan doses and the measured plan doses by specific quality assurance (QA) devices and is usually used in RT clinics for QA of IMRT and VMAT plans $[22,23]$. However, in our study, we applied this concept to compare the two plans and to show the dose differences as a single numeric. A two-dimensional gamma index measured by the electronic portal imaging device (EPID) and calculated by the Varian portal dosimetry system (version 13.6) was used to quantitatively evaluate the changes in the actual delivered dose arising due to WT changes. We adopted the equation for gamma index suggested by Low et al. [23] and modified it as follows:

$$
\text { Gamma index }=\min \left\{\Gamma\left(r_{\mathrm{e}}, r_{\mathrm{s}}\right)\right\} \quad \forall\left\{r_{\mathrm{s}}\right\}
$$

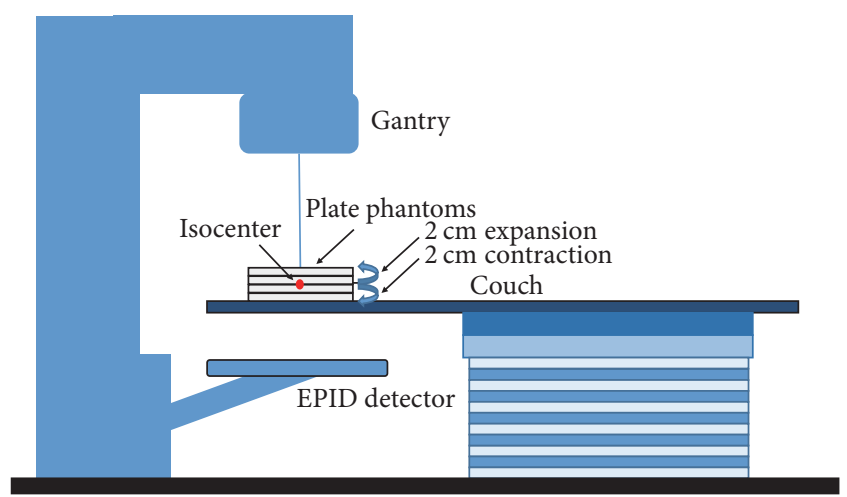

Figure 2: A simplified illustration to show the gamma index evaluation method.

where $r_{\mathrm{e}}$ is a single measurement point in the experimental plan (with WT change) and $r_{\mathrm{s}}$ is a single measurement point in the standard plan (no WT change).

$$
\begin{aligned}
& \Gamma\left(r_{\mathrm{e}}, r_{\mathrm{s}}\right)=\sqrt{\frac{r^{2}\left(r_{\mathrm{e}}, r_{\mathrm{s}}\right)}{\Delta d^{2}}+\frac{\delta^{2}\left(r_{\mathrm{e}}, r_{\mathrm{s}}\right)}{\Delta D^{2}}} \\
& r\left(r_{\mathrm{e}}, r_{\mathrm{s}}\right)=\left|r_{\mathrm{s}}-r_{\mathrm{e}}\right|, \\
& \delta\left(r_{\mathrm{e}}, r_{\mathrm{s}}\right)=D_{\mathrm{s}}\left(r_{\mathrm{s}}\right)-D_{\mathrm{e}}\left(r_{\mathrm{e}}\right),
\end{aligned}
$$

where $\Delta d$ and $\Delta D$ are the distance and dose passing criteria which were defined as $3 \mathrm{~mm}$ and $3 \%$ in this study, while $D_{x}$ is the measured dose in the "s" (standard) or " $\mathrm{e}$ " (experimental) plans. The point is passed if the gamma value is 1 or below 1 , and the point is failed if the gamma value is higher than 1 . The gamma passing rate is defined as follows:

$$
\begin{aligned}
& \text { Gamma passing rate } \\
& =\frac{\text { the number of passed points }(\text { gamma }<1)}{\text { the number of all points }} .
\end{aligned}
$$

The experimental plan is regarded as unacceptable if the gamma passing rate is below $95 \%$ or the maximum gamma index is higher than 3.5.

For this measurement, we used 0.5 and $1 \mathrm{~cm}$ thick plate phantoms to reflect the patients' body contour changes $(-2.0 \mathrm{~cm}$ to $2.0 \mathrm{~cm})$. A VMAT plan with the same monitor unit (MU) and gantry rotation was employed to irradiate these phantoms with varied thicknesses (Figure 2). The dose distribution measured using the $2 \mathrm{~cm}$ thick phantom was regarded as the standard (no WT change). This was compared to the dose distributions measured using phantoms with a thickness ranging from 0 to $4 \mathrm{~cm}$. An example of the gamma index analysis result is shown in Figure 3.

\section{Results}

3.1. Target Coverage. The results of the dosimetric analysis of target coverage are shown in Table 1 and Figures 4(a)4(b). The mean $D_{95 \%}$ for PTV decreased gradually as body 


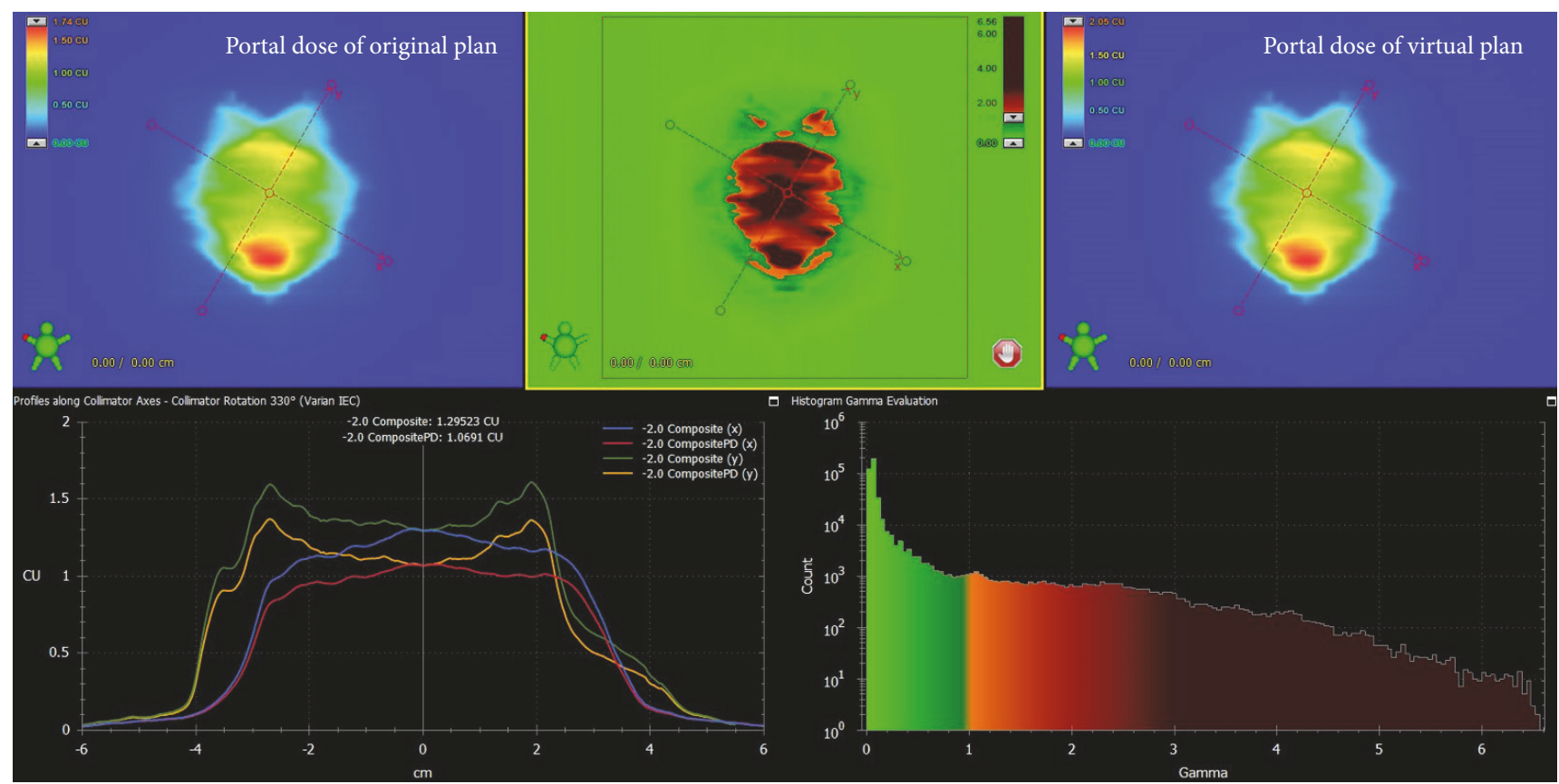

Figure 3: A gamma index analysis with the $3 \mathrm{~mm} / 3 \%$ criterion for comparing the original plan and experimental plan using a $-2.0 \mathrm{~cm}$ contracted body contour. The number of points falling beyond our gamma criteria is represented by the red spots in the middle image.

TABLE 1: The changes in dosimetric parameters for (a) planning target volume coverage and (b) conformity and homogeneity indices with changes in body contour from $-2.0 \mathrm{~cm}$ to $2.0 \mathrm{~cm}$.

(a) Planning target volume coverage (\%)

\begin{tabular}{ccccccccccc}
\hline & & \multicolumn{9}{c}{ Body contour changes $(\mathrm{cm})$} \\
& & -2.0 & -1.5 & -1.0 & -0.5 & 0 & 0.5 & 1.0 & 1.5 & 2.0 \\
\hline \multirow{2}{*}{$6-\mathrm{MV}$} & $D_{95 \%}$ & 103.08 & 102.33 & 101.49 & 100.66 & 100.00 & 99.28 & 98.31 & 97.35 & 96.34 \\
& $D_{\max }$ & 110.74 & 108.94 & 107.34 & 106.06 & 105.36 & 104.72 & 104.06 & 103.52 & 103.00 \\
$10-\mathrm{MV}$ & $D_{95 \%}$ & 102.83 & 101.83 & 101.13 & 100.51 & 100.00 & 99.48 & 98.81 & 98.04 & 97.39 \\
& $D_{\max }$ & 108.78 & 107.50 & 106.32 & 105.48 & 105.02 & 104.68 & 104.06 & 103.56 & 103.12 \\
$15-\mathrm{MV}$ & $D_{95 \%}$ & 102.22 & 101.66 & 101.05 & 100.46 & 100.00 & 99.52 & 98.93 & 98.30 & 97.68 \\
& $D_{\max }$ & 108.72 & 107.56 & 106.50 & 105.62 & 105.12 & 104.60 & 104.02 & 103.54 & 103.12 \\
\hline
\end{tabular}

$D_{x \%}$ : the percentage of the prescription dose covering $x \%$ of the volume. Italic font: unacceptable.

(b) Conformity and homogeneity indices

\begin{tabular}{lcccccccccc}
\hline & & \multicolumn{9}{c}{ Body contour changes $(\mathrm{cm})$} \\
& & -2.0 & -1.5 & -1.0 & -0.5 & 0 & 0.5 & 1.0 & 1.5 & 2.0 \\
\hline \multirow{2}{*}{$6-\mathrm{MV}$} & $\mathrm{CI}$ & 1.18 & 1.14 & 1.10 & 1.05 & 1.00 & 0.91 & 0.62 & 0.33 \\
& $\mathrm{HI}$ & 1.047 & 1.037 & 1.032 & 1.029 & 1.028 & 1.029 & 1.033 & 1.037 & 1.043 \\
\multirow{2}{*}{$10-\mathrm{MV}$} & $\mathrm{CI}$ & 1.15 & 1.11 & 1.08 & 1.03 & 1.00 & 0.93 & 0.74 & 0.50 & 0.31 \\
& $\mathrm{HI}$ & 1.040 & 1.039 & 1.029 & 1.028 & 1.027 & 1.028 & 1.030 & 1.033 & 1.036 \\
& $\mathrm{CI}$ & 1.14 & 1.11 & 1.07 & 1.02 & 0.99 & 0.94 & 0.78 & 0.55 & 0.39 \\
& HI & 1.042 & 1.037 & 1.033 & 1.031 & 1.030 & 1.026 & 1.031 & 1.033 & 1.034 \\
\hline
\end{tabular}

$\mathrm{CI}$, conformity index; HI, homogeneity index.

contour expanded from $-2.0 \mathrm{~cm}$ to $2 \mathrm{~cm}$ by $0.5 \mathrm{~cm}$ increments. Among the three different photon energy plans, the 6MV plan showed the most prominent decrease. $D_{95 \%}$ became lower than $98 \%$ when the body contours were expanded to $1.5 \mathrm{~cm}$ and $2.0 \mathrm{~cm}$ in the 6-MV and 10-MV plans, respectively.
The mean $D_{\max }$ for PTV increased as the body contour contracted and decreased as the body contour expanded. $D_{\max }$ for PTV became higher than $107 \%$ when the body contour was contracted by $1.5 \mathrm{~cm}$ or more in the $10-\mathrm{MV}$ plans and by $1.0 \mathrm{~cm}$ or more in the $6-\mathrm{MV}$ plans. The mean CI also 


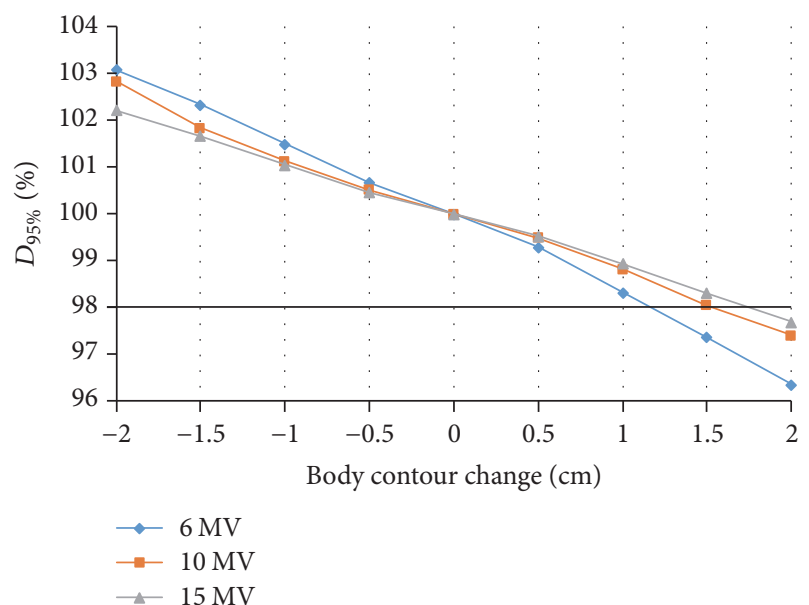

(a)

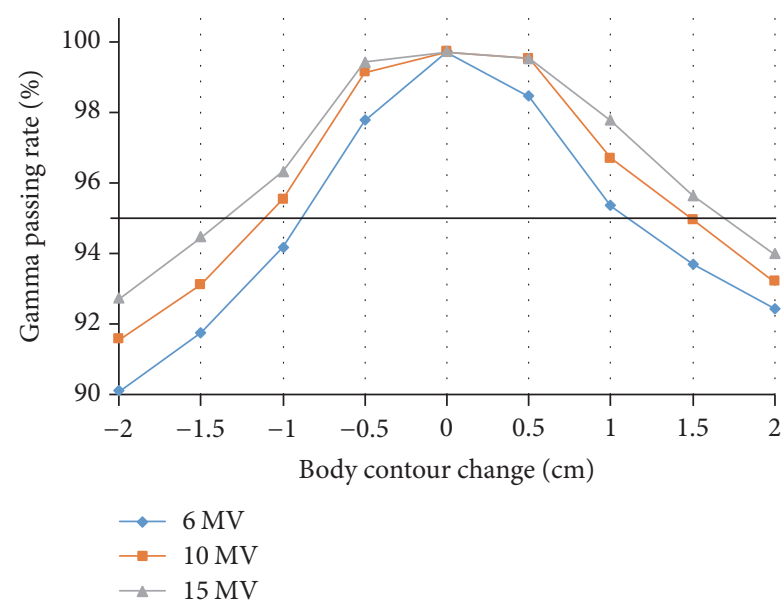

(c)

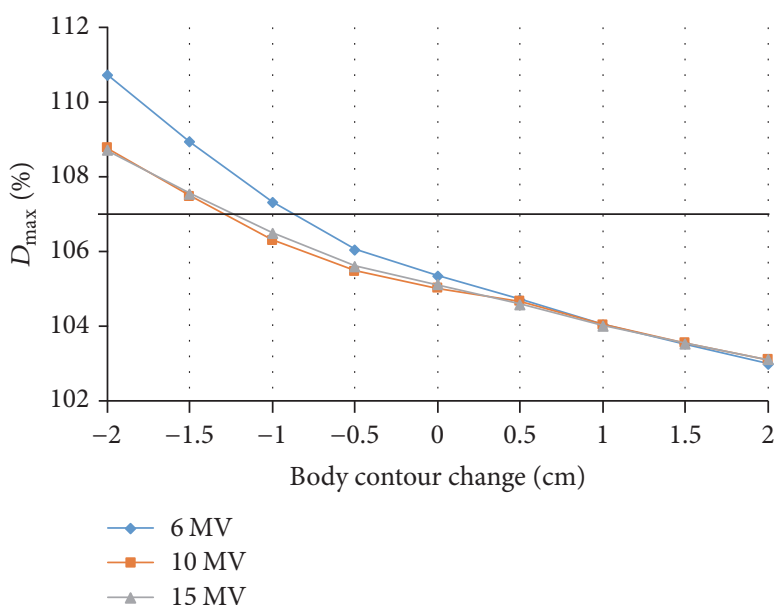

(b)

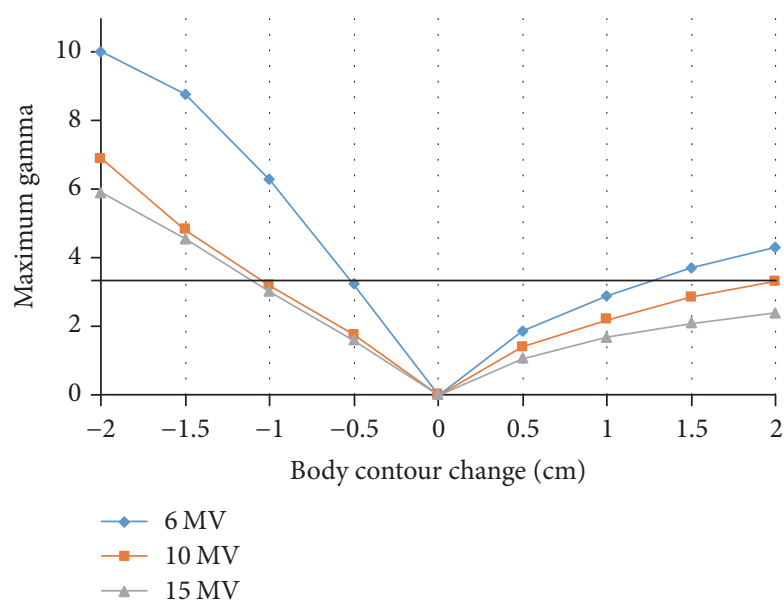

(d)

FIgURE 4: The changes in dosimetric parameters (a) $D_{95 \%}$ and (b) $D_{\max }$ for the PTV and the changes in (c) gamma passing rates and (d) maximum gamma values with changes in body contour for different photon energy levels. The solid lines are the acceptable threshold lines.

decreased gradually as the body contour expanded. The CI was lower than 0.5 when the body contour was expanded by $1.5 \mathrm{~cm}$ and $2.0 \mathrm{~cm}$ in the 6-MV and 10-MV plans, respectively. The mean HI for PTV deteriorated as the body contour was contracted or expanded. The results of the VMAT plans using 15-MV photon energy were similar to those using 10-MV photon energy.

3.2. Normal Tissue Sparing. The dose to the rectum and bladder increased gradually as the body contour was contracted and decreased as the body contour was expanded. Among the three different photon energy plans, the 6-MV plan showed the most prominent decrease. The rectum $V_{70}$ Gy increased from $9.57 \%$ to $10.67 \%$ as the body contour was contracted by $2.0 \mathrm{~cm}$ in the $10-\mathrm{MV}$ plans. The bladder $V_{70 \mathrm{~Gy}}$ also increased from $13.59 \%$ to $14.61 \%$ in the $10-\mathrm{MV}$ plans. However, these differences were relatively small and all parameters satisfied the normal tissue dose constraints even in the 6-MV plans with body contours contracted by $2.0 \mathrm{~cm}$. Overall the detailed dose-volume data for $V_{70 \text { Gy }}$ for the rectum and bladder are shown in Table 2 and Figure 5. Figure 6 shows changes in the $\mathrm{DVH}$ of one patient.
3.3. Gamma Index Analysis. Table 3 and Figures 4(c)-4(d) show the changes in gamma passing rates and the maximum gamma index. The mean gamma passing rates decreased in both directions as the body contours were expanded or contracted. In the 6-MV plans, the gamma passing rates became lower than $95 \%$ both when the body contour was contracted by $1.0 \mathrm{~cm}$ or more and when the body contour was expanded by $1.5 \mathrm{~cm}$ or more. The maximum gamma index was also higher than 3.5 under the same conditions. In the 10-MV and 15-MV plans, the gamma index analysis showed unacceptable results when the body contour was either contracted by $1.5 \mathrm{~cm}$ or more or expanded by $2.0 \mathrm{~cm}$ or more.

\section{Discussion}

What Can We Do If the Body Contour Change Impedes the Accuracy of Dose Delivery? Since individual body contour changes cannot be accounted for by simple geometric correction methods, such as target position corrections by couch or patient shifts, other strategies should be considered. Some of 
TABLE 2: The changes in dosimetric parameters for the rectum (R) and bladder (B) with the changes of body contour from $-2.0 \mathrm{~cm}$ to $2.0 \mathrm{~cm}$.

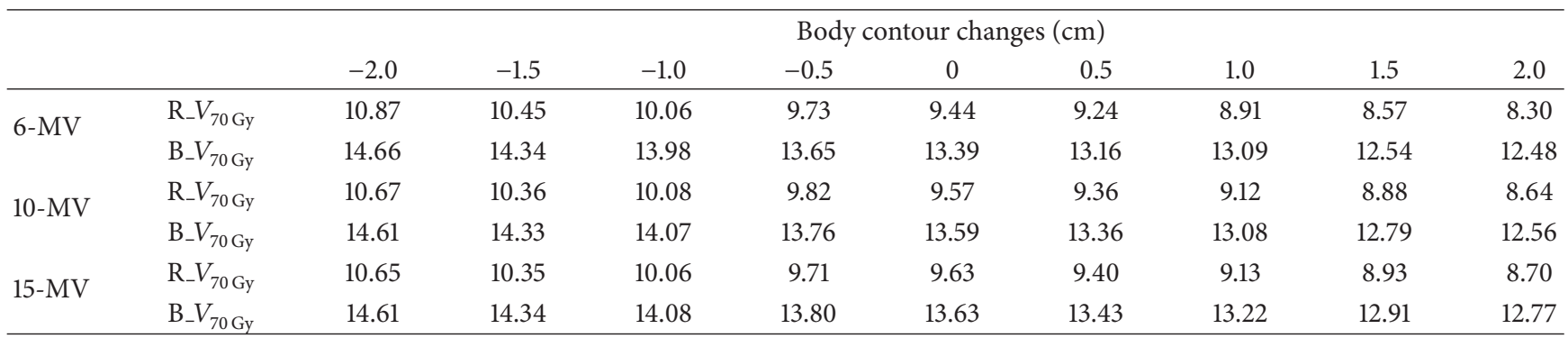

$V_{x \mathrm{~Gy}}$, the percentage of the organ volume receiving $x$ Gy or more; $\mathrm{R}_{-} V_{70 \mathrm{~Gy}}$, rectum $V_{70 \mathrm{~Gy}} ; \mathrm{B}_{-} V_{70 \mathrm{~Gy}}$, bladder $V_{70 \mathrm{~Gy}}$.

TABle 3: The changes in gamma index with changes in body contour from $-2.0 \mathrm{~cm}$ to $2.0 \mathrm{~cm}$.

\begin{tabular}{llccccccccc}
\hline & & & \multicolumn{9}{c}{ Body contour changes $(\mathrm{cm})$} \\
& & -2.0 & -1.5 & -1.0 & -0.5 & 0 & 0.5 & 1.0 & 1.5 & 2.0 \\
\hline \multirow{2}{*}{$6-\mathrm{MV}$} & GPR & 90.10 & 91.80 & 94.30 & 98.00 & 100.00 & 98.70 & 95.50 & 93.80 & 92.50 \\
& MG & 10.00 & 8.76 & 6.27 & 3.21 & 0.00 & 1.85 & 2.88 & 3.69 & 4.29 \\
\multirow{2}{*}{$10-\mathrm{MV}$} & GPR & 91.60 & 93.20 & 95.70 & 99.40 & 100.00 & 99.80 & 96.90 & 95.10 & 93.30 \\
& MG & 6.89 & 4.81 & 3.19 & 1.75 & 0.00 & 1.39 & 2.19 & 2.85 & 3.30 \\
& GPR & 92.80 & 94.60 & 96.50 & 99.70 & 100.00 & 99.80 & 98.00 & 95.80 & 94.10 \\
& MG & 5.90 & 4.55 & 3.01 & 1.58 & 0.00 & 1.06 & 1.68 & 2.08 & 2.38 \\
\hline
\end{tabular}

GPR, gamma pass rate; MG, maximum gamma values. Italic font: unacceptable.

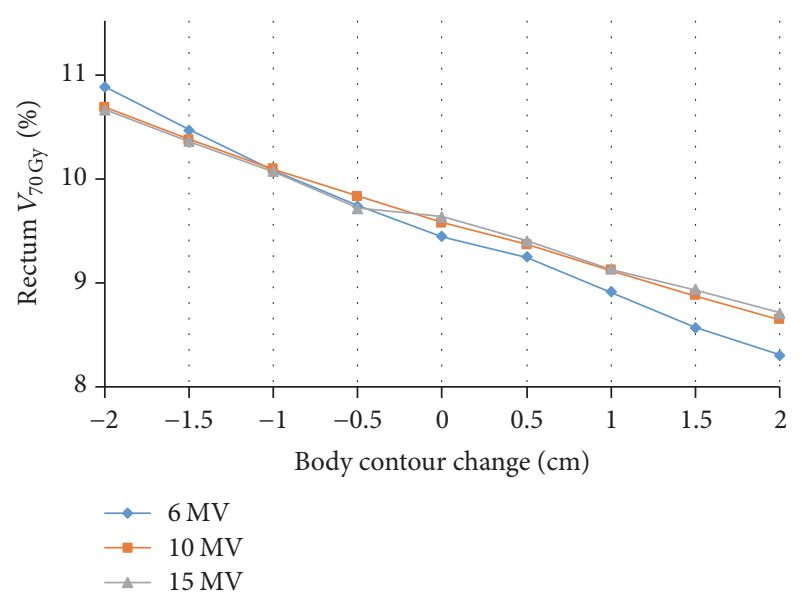

(a)

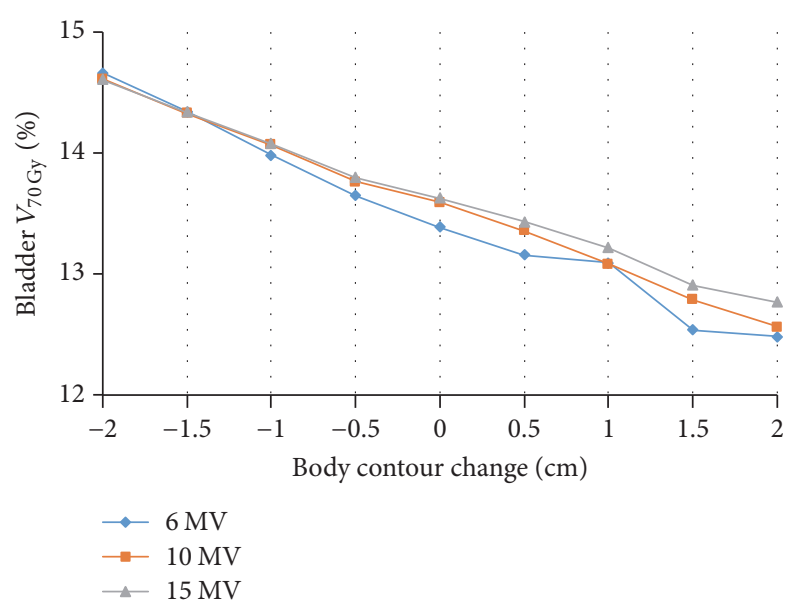

(b)

FIGURE 5: The changes in dosimetric parameters (a) rectum $V_{70 \text { Gy }}$ and (b) bladder $V_{70 \text { Gy }}$ with changes in body contour from $-2.0 \mathrm{~cm}$ to $2.0 \mathrm{~cm}$ for different photon energy levels.

these strategies include (a) using large PTV margins [24, 25] to cover various body contour changes, (b) tolerating the risk of over- or undertreating the target, or (c) replanning the treatment so as to adapt to the individual body changes $[26,27]$. Ethically, ART is probably the most reasonable solution to date. Recently, Castelli et al. [28] have studied the benefits of adaptive replanning in head and neck cancers with frequent anatomical variations such as weight change and tumor shrinkage. They reported that weekly replanning could reduce the mean dose to the parotid glands by $5.1 \mathrm{~Gy}$. This dose difference could reduce the absolute risk of xerostomia by an average of $11 \%$. In our study on the prostate cancer, the adaptive replanning according to weight change had the benefits of increased $D_{95 \%}$ of the target volume up to $2.61 \%$ and reduced $D_{\max }$ of the target volume, rectum $V_{70 \mathrm{~Gy}}$, and bladder $V_{70 \text { Gy }}$ up to $3.76 \%, 1.1 \%$, and $1.02 \%$, respectively. However, despite this benefit there are no definite criteria when ART should be considered using body contour or WT changes.

When Should ART Be Considered? To the best of our knowledge, no studies have been conducted to address this question. In our study, as body contours changed $(-2 \mathrm{~cm}$ to $2 \mathrm{~cm}$ ), the dose delivered to the normal tissues did not 


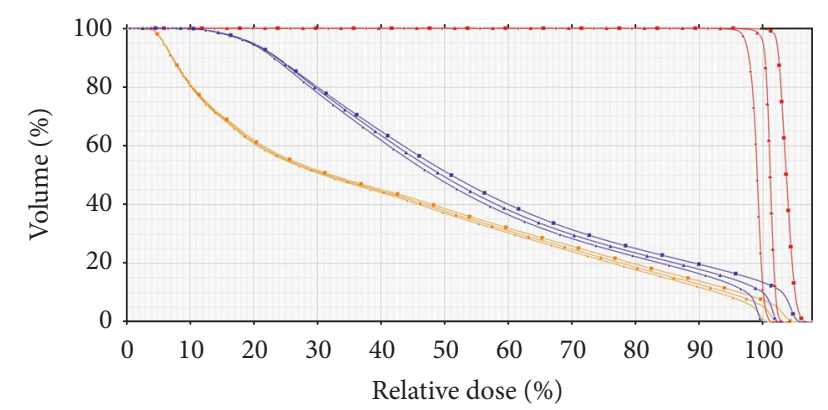

FIgURE 6: An example of a dose-volume histograms (DVH) of the volumetric modulated arc radiotherapy plan using $10-\mathrm{MV}$ photon energy for a prostate cancer patient. The dose-volume histograms are indicated using the different colors (PTV, red; rectum, orange; bladder, blue) and types of lines $(-2.0 \mathrm{~cm}$ body contour, square; original body contour, triangle; $2.0 \mathrm{~cm}$ body contour, small circle).

cross the tolerance limit. However, the dosimetric parameters and gamma index analysis yielded consistent results for target coverage. With the use of 6-MV photon energy, the plan became unacceptable if the body contour was either contracted by $1 \mathrm{~cm}$ or more or expanded by $1.5 \mathrm{~cm}$ or more. However, with $10-\mathrm{MV}$ or $15-\mathrm{MV}$ photon energies, a contraction by $1.5 \mathrm{~cm}$ or expansion by $2.0 \mathrm{~cm}$ made the plan unacceptable. Therefore, ART should be considered if the depth of body contour becomes smaller than $1.5 \mathrm{~cm}$ or larger than $2.0 \mathrm{~cm}$. Changes beyond these values could affect the target coverage and cause a failure in the gamma index analysis. The depth of body contour could be measured by acquiring in-room $\mathrm{kV}$ or MV cone-beam CT (CBCT) images or by simply measuring the source-to-skin distance (SSD) according to the equipment of each institution. However, when using only SSD, several factors should be considered, such as patient positioning and immobilization.

What Is the Optimal Photon Energy for Prostate Cancer Patients? Several studies have reported better dosimetric distribution with 10-MV plans compared to 6-MV plans for IMRT and VMAT $[28,29]$. Plans with 15-MV have little advantage over the 10-MV plans but may carry an increased risk of neutron contamination $[29,30]$. Our results demonstrate that the 10-MV plans are better than the 6-MV plans. The 10-MV plans resulted in smaller dose differences as the body contour or WT changed, compared to the 6MV plans. However, since the differences between the 10MV and 15-MV plans were relatively small and the risk of neutron contamination increased for the higher energy doses, to use the 10-MV photon beams for IMRT of prostate cancer is recommended.

Limitations. First, our study was limited to VMAT plans, treating only the prostate and seminal vesicles. Although the targets and OAR were contoured following the guidelines of RTOG, several planning factors such as the optimization skills could alter the study results. Therefore, our findings and the suggested cut-off values might not be applicable to all institutions. The second limitation is that, in the actual clinical cases, body contours do not change in a uniform manner as shown in Figure 1. In addition, the position of the internal organs could change as the body WT changes. However, these factors were not considered in this study. Further retrospective studies using WT changes from real clinical cases are needed to answer these questions. However, our study presents a guideline for the choosing the optimal time to give ART to prostate cancer patients with WT changes.

\section{Conclusion}

In conclusion, we have found that WT changes during prostate VMAT can cause considerable change in the target dose distribution. We suggest that when using 10-MV VMAT plans for patients with prostate cancer, the appropriate time to consider adaptive replanning is when the body contour becomes $1.5 \mathrm{~cm}$ smaller (WT loss) or $2.0 \mathrm{~cm}$ larger (WT gain) than the original value.

\section{Conflicts of Interest}

The authors declare that they have no conflicts of interest regarding the publication of this paper.

\section{Authors' Contributions}

Hoon Sik Choi and Guang Sub Jo contributed equally to this work.

\section{References}

[1] L. Feuvret, G. Noël, C. Nauraye, P. Garcia, and J.-J. Mazeron, "Conformal index and radiotherapy," Cancer Radiothérapie, vol. 8, no. 2, pp. 108-119, 2004.

[2] M. J. Zelefsky, Z. Fuks, L. Happersett et al., "Clinical experience with intensity modulated radiation therapy (IMRT) in prostate cancer," Radiotherapy \& Oncology, vol. 55, no. 3, pp. 241-249, 2000.

[3] D. Palma, E. Vollans, K. James et al., "Volumetric modulated arc therapy for delivery of prostate radiotherapy: comparison with intensity-modulated radiotherapy and three-dimensional conformal radiotherapy," International Journal of Radiation Oncology Biology Physics, vol. 72, no. 4, pp. 996-1001, 2008.

[4] H. J. Kang, C.-S. Kay, S. H. Son et al., "Hypofractionated intensity-modulated radiotherapy in patients with localized prostate cancer: a preliminary study," Radiation Oncology Journal, vol. 34, no. 1, pp. 45-51, 2016.

[5] Y. S. Kim, J. Lee, J. I. Park, W. Sung, S. M. Lee, and G. E. Kim, "Volumetric modulated arc therapy for carotid sparing in the management of early glottic cancer," Radiation Oncology Journal, vol. 34, no. 1, pp. 18-25, 2016.

[6] M. J. Zelefsky, M. Kollmeier, B. Cox et al., "Improved clinical outcomes with high-dose image guided radiotherapy compared with non-IGRT for the treatment of clinically localized prostate cancer," International Journal of Radiation Oncology Biology Physics, vol. 84, no. 1, pp. 125-129, 2012.

[7] W. Y. Song, B. Schaly, G. Bauman, J. J. Battista, and J. Van Dyk, "Evaluation of image-guided radiation therapy (IGRT) technologies and their impact on the outcomes of hypofractionated prostate cancer treatments: a radiobiologic analysis," 
International Journal of Radiation Oncology Biology Physics, vol. 64, no. 1, pp. 289-300, 2006.

[8] J. Nijkamp, F. J. Pos, T. T. Nuver et al., "Adaptive radiotherapy for prostate cancer using kilovoltage cone-beam computed tomography: first clinical results," International Journal of Radiation Oncology Biology Physics, vol. 70, no. 1, pp. 75-82, 2008.

[9] D. Yan, D. Lockman, D. Brabbins, L. Tyburski, and A. Martinez, "An off-line strategy for constructing a patient-specific planning target volume in adaptive treatment process for prostate cancer," International Journal of Radiation Oncology Biology Physics, vol. 48, no. 1, pp. 289-302, 2000.

[10] D. L. Schwartz, A. S. Garden, J. Thomas et al., "Adaptive radiotherapy for head-and-neck cancer: initial clinical outcomes from a prospective trial," International Journal of Radiation Oncology Biology Physics, vol. 83, no. 3, pp. 986-993, 2012.

[11] R. L. Siegel, K. D. Miller, and A. Jemal, "Cancer statistics, 2017," CA: A Cancer Journal for Clinicians, vol. 67, no. 1, pp. 7-30, 2017.

[12] A. V. D’Amico, R. Whittington, S. Bruce Malkowicz et al., "Biochemical outcome after radical prostatectomy, external beam radiation therapy, or interstitial radiation therapy for clinically localized prostate cancer," Journal of the American Medical Association, vol. 280, no. 11, pp. 969-974, 1998.

[13] W. D. D'Souza and H. D. Thames, "Is the $\alpha / \beta$ ratio for prostate cancer low?" International Journal of Radiation Oncology Biology Physics, vol. 51, no. 1, pp. 1-3, 2001.

[14] B.-K. Jeong, H. Jeong, I. B. Ha et al., "Stereotactic body radiation therapy for low- to intermediaterisk prostate adenocarcinoma," Journal of Korean Medical Science, vol. 30, no. 6, pp. 710-715, 2015.

[15] M. R. Smith, J. S. Finkelstein, F. J. McGovern et al., "Changes in body composition during androgen deprivation therapy for prostate cancer," The Journal of Clinical Endocrinology \& Metabolism, vol. 87, no. 2, pp. 599-603, 2002.

[16] J. C. L. Chow and R. Jiang, "Comparison of dosimetric variation between prostate IMRT and VMAT due to patient's weight loss: patient and phantom study," Reports of Practical Oncology and Radiotherapy, vol. 18, no. 5, pp. 272-278, 2013.

[17] L. B. Marks, E. D. Yorke, A. Jackson et al., "Use of normal tissue complication probability models in the clinic," International Journal of Radiation Oncology Biology Physics, vol. 76, no. 3, pp. S10-S19, 2010.

[18] I. J. Das, A. Andersen, Z. J. Chen et al., "State of dose prescription and compliance to international standard (ICRU83) in intensity modulated radiation therapy among academic institutions," Practical Radiation Oncology, vol. 7, no. 2, pp. e145e155, 2017.

[19] V. Chanyavanich, S. K. Das, W. R. Lee, and J. Y. Lo, "Knowledgebased IMRT treatment planning for prostate cancer," Medical Physics, vol. 38, no. 5, pp. 2515-2522, 2011.

[20] L. Feuvret, G. Noël, J.-J. Mazeron, and P. Bey, "Conformity index: a review," International Journal of Radiation Oncology Biology Physics, vol. 64, no. 2, pp. 333-342, 2006.

[21] D. Saenz, B. Paliwal, and J. Bayouth, "A dose homogeneity and conformity evaluation between ViewRay and pinnacle-based linear accelerator IMRT treatment plans," Journal of Medical Physics, vol. 39, no. 2, pp. 64-70, 2014.

[22] J. Song, M. Kim, S. Park et al., "Gamma analysis dependence on specified low-dose thresholds for VMAT QA," Journal of Applied Clinical Medical Physics, vol. 16, no. 6, pp. 263-272, 2015.

[23] D. A. Low, W. B. Harms, S. Mutic, and J. A. Purdy, "A technique for the quantitative evaluation of dose distributions," Medical Physics, vol. 25, no. 5, pp. 656-661, 1998.
[24] E. Meldolesi, D. Lockman, D. Yan, and A. Martinez, "Geometric evaluation of image guided adaptive radiotherapy (ART) for prostate cancer," International Journal of Radiation Oncology Biology Physics, vol. 63, pp. S90-S91, 2005.

[25] D. Yan, F. Vicini, J. Wong, and A. Martinez, "Adaptive radiation therapy," Physics in Medicine and Biology, vol. 42, no. 1, pp. 123132, 1997.

[26] R. Mohan, X. Zhang, H. Wang et al., "Use of deformed intensity distributions for on-line modification of image-guided IMRT to account for interfractional anatomic changes," International Journal of Radiation Oncology Biology Physics, vol. 61, no. 4, pp. 1258-1266, 2005.

[27] E. E. Ahunbay, C. Peng, S. Holmes, A. Godley, C. Lawton, and X. A. Li, "Online adaptive replanning method for prostate radiotherapy," International Journal of Radiation Oncology Biology Physics, vol. 77, no. 5, pp. 1561-1572, 2010.

[28] J. Castelli, A. Simon, G. Louvel et al., "Impact of head and neck cancer adaptive radiotherapy to spare the parotid glands and decrease the risk of xerostomia," Journal of Radiation Oncology, vol. 10, no. 1, article 6, 2015.

[29] W. Sung, J. M. Park, C. H. Choi, S. W. Ha, and S.-J. Ye, “The effect of photon energy on intensity-modulated radiation therapy (IMRT) plans for prostate cancer," Radiation Oncology Journal, vol. 30, no. 1, pp. 27-35, 2012.

[30] D. N. Stanley, T. Popp, C. S. Ha et al., "Dosimetric effect of photon beam energy on volumetric modulated arc therapy treatment plan quality due to body habitus in advanced prostate cancer," Practical Radiation Oncology, vol. 5, no. 6, pp. e625e633, 2015. 


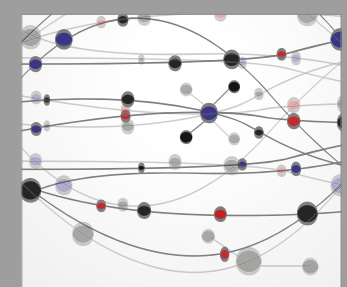

The Scientific World Journal
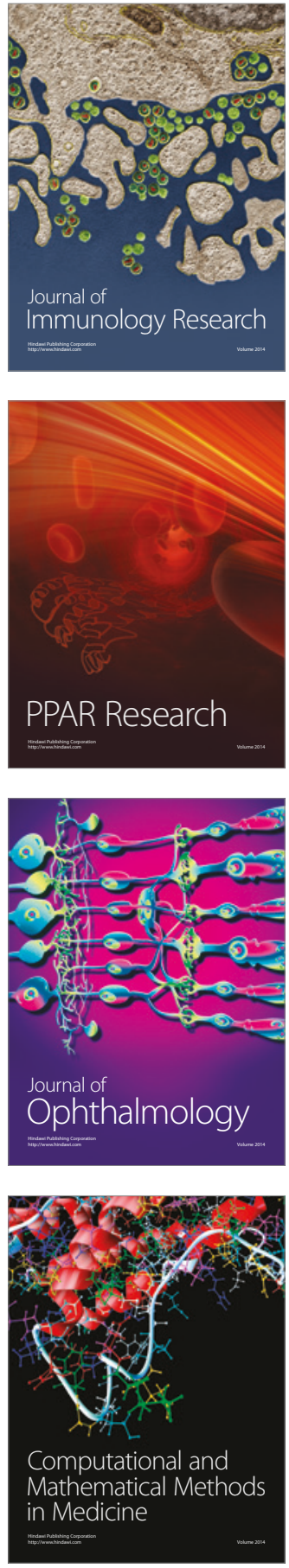

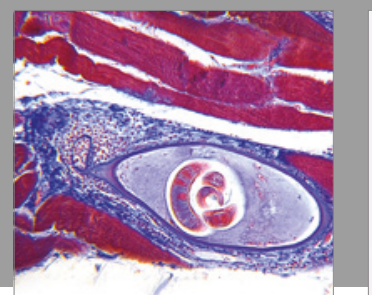

Gastroenterology Research and Practice
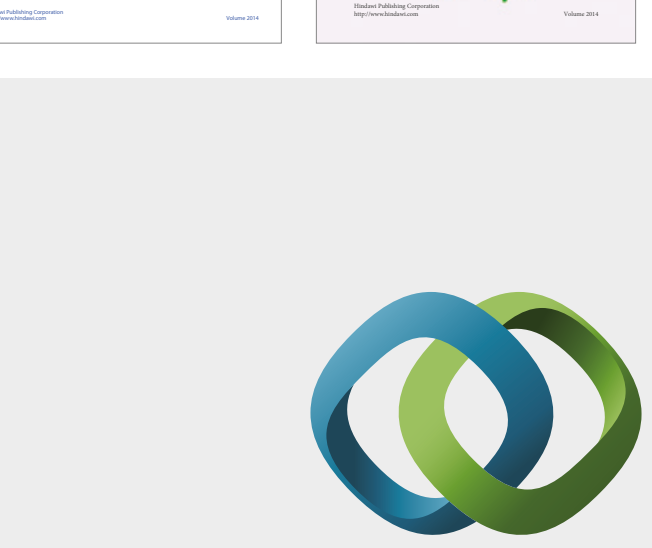

\section{Hindawi}

Submit your manuscripts at

https://www.hindawi.com
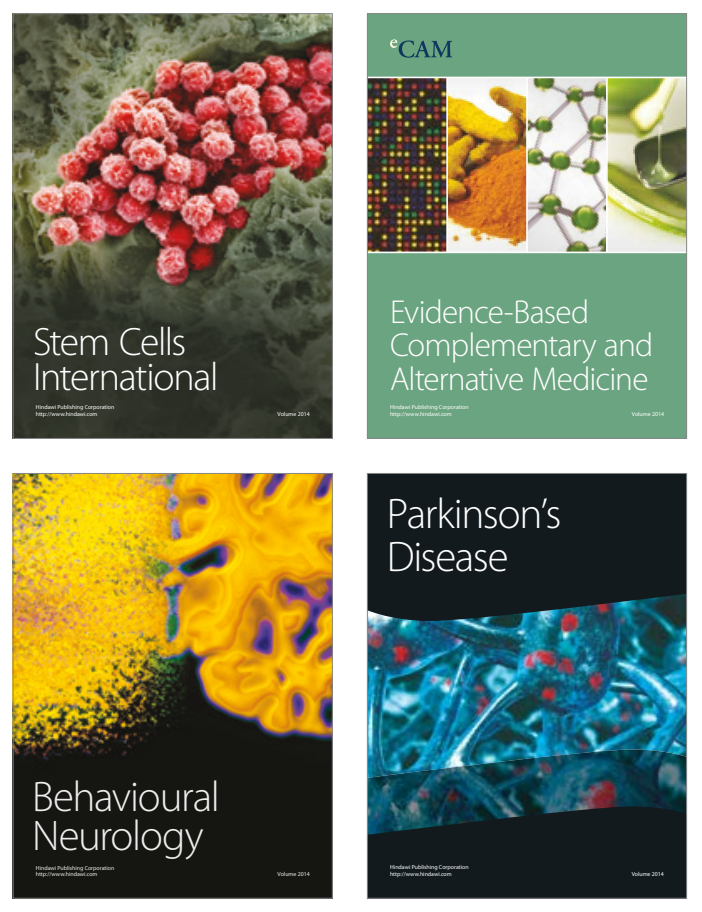
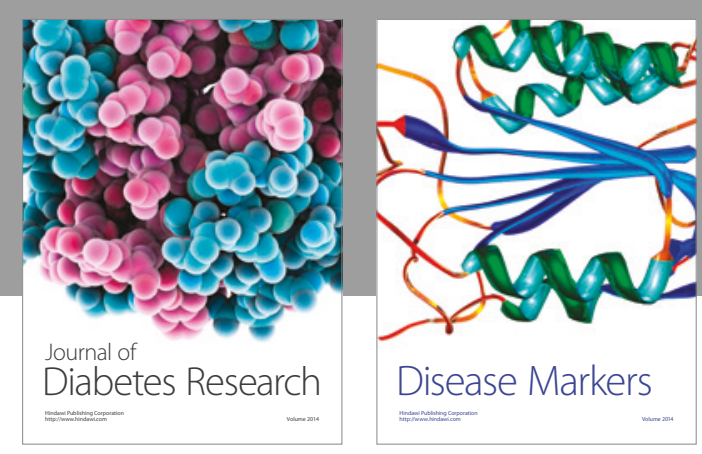

Disease Markers
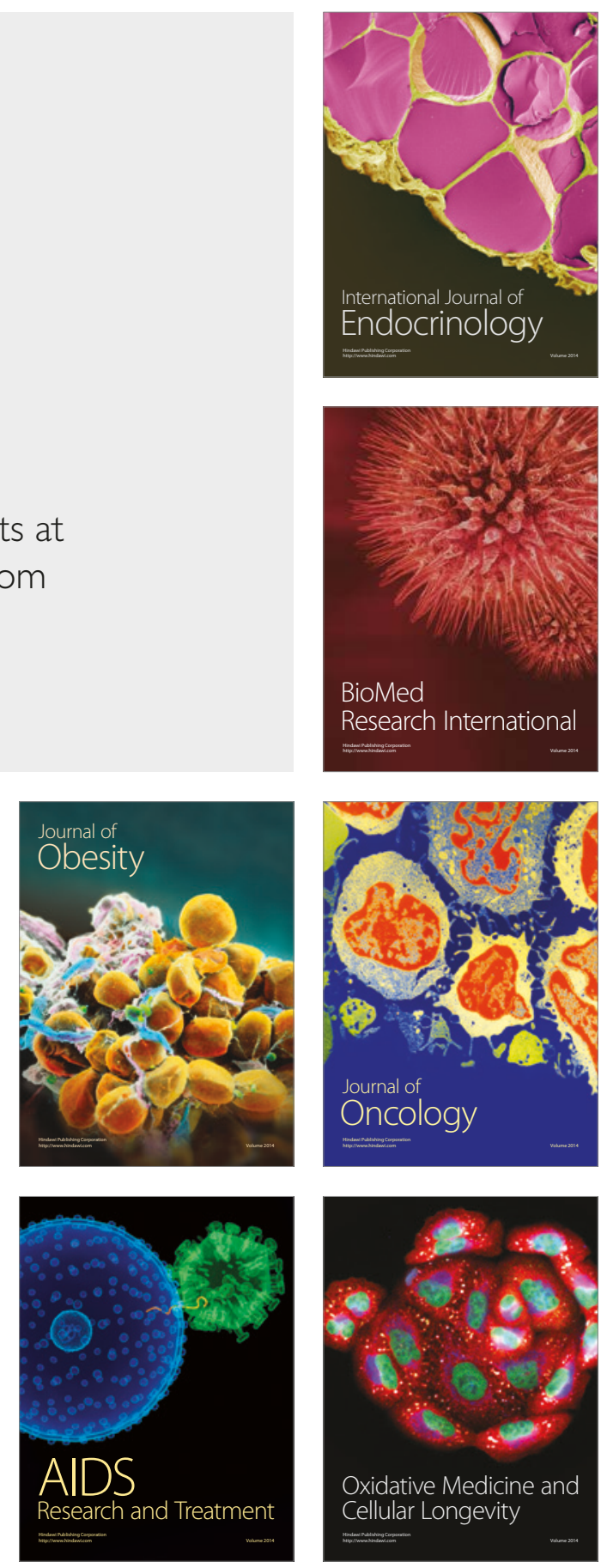